\title{
Diagnosis and treatment of post-traumatic hypothermia in hospitals - a pilot study
}

Authors' Contribution: A-Study Design B-Data Collection C-Statistical Analysis D-Data Interpretation E-Manuscript Preparation F-Literature Search G-Funds Collection

\author{
Paweł Podsiadło ${ }^{1,2 A B D E F}$, Sylweriusz Kosiński, ${ }^{3,4 D E F}$, Tomasz Darocha ${ }^{2,5 D E F}$, Katherine Zerebiec, ${ }^{6,7 E F}$, \\ Przemysław Wolak ${ }^{8,98 E}$, Stanislas Kielczewski ${ }^{\mathrm{EF}}$, Sławomir Plewa ${ }^{10 \mathrm{DE}}$, Stanisław Cłuszek ${ }^{11,12 \mathrm{DE}}$ \\ 'Department of Emergency Medicine, Jan Kochanowski University, Kielce, Poland \\ 2Polish Medical Air Rescue, Warsaw, Poland \\ ${ }^{3}$ Jagiellonian University Medical College, Faculty of Health Sciences, Krakow, Poland \\ ${ }^{4}$ Tatra Mountain Rescue Service, Zakopane, Poland \\ ${ }^{5}$ Department of Anesthesiology and Intensive Care, Medical University of Silesia, Katowice, Poland \\ ${ }^{6}$ Wilderness Medical Society, Salt Lake City, USA \\ ${ }^{7}$ Student of Jagiellonian University Medical College, Krakow, Poland \\ ${ }^{8}$ Department of Pediatric Surgery, Urology, and Traumatology, Regional Hospital, Kielce, Poland \\ ${ }^{9}$ Department of Pediatrics, Pediatric and Social Nursing, Institute of Nursing and Midwifery, Faculty of Medicine and Health Scien- \\ ces, Jan Kochanowski University, Kielce, Poland \\ ${ }^{10} \mathrm{Clinic}$ of Anesthesiology and Intensive Care, Regional Hospital, Kielce, Poland \\ "Department of Surgery and Surgical Nursing with Research and Cenetic Laboratory, Faculty of Medicine and Health Sciences, Jan \\ Kochanowski University, Kielce, Poland \\ ${ }^{12}$ Clinical Department of Ceneral, Oncological and Endocrinological Surgery, Regional Hospital, Kielce, Poland
}

Article history: Received: 07.08.2018 Accepted: 21.01.2019 Published: 07.02.2019

ABSTRACT: $\quad$ Background: An unintentional drop in core body temperature of trauma victims is associated with increased mortality. Thermoregulation is impaired in these patients, especially when treated with opioids or anesthetics. Careful thermal insulation and active warming are necessary to maintain normothermia. The aim of the study was to assess the equipment and procedures for diagnosing and managing post-traumatic hypothermia in Polish hospitals.

Methods: Survey forms regarding equipment and procedures on monitoring of core temperature (Tc) and active warming were distributed to every hospital that admits trauma victims in the Holy Cross Province. Questionnaires were addressed to surgery departments, intensive care units (ICUs), and operating rooms (ORs).

Results: $92 \%$ of surgery departments did not have equipment to measure core body temperature and $85 \%$ did not have equipment to rewarm patients. Every ICU had equipment to measure Tc and $83 \%$ had active warming devices. In $50 \%$ of ICUs, there were no rewarming protocols based on Tc and the initiation of rewarming was left to the physician's discretion. In $58 \%$ of ORs, Tc was not monitored and in $33 \%$ the patients were not actively warmed.

Conclusions: The majority of surveyed ICUs and ORs are adequately equipped to identify and treat hypothermia, however the criteria for initiating Tc monitoring and rewarming remain unstandardized. Surgery departments are not prepared to manage post-traumatic hypothermia.

KEYWORDS: $\quad$ trauma, hypothermia, resuscitation, emergency medicine, rewarming

\section{INTRODUCTION}

There are three stages of post-traumatic hypothermia: mild $\left(36-34^{\circ} \mathrm{C}\right)$, moderate $\left(34-32^{\circ} \mathrm{C}\right)$, and severe $\left(<32^{\circ} \mathrm{C}\right)$. This classification differs from accidental hypothermia due to the significant impact of injury-associated hypothermia on mortality [1]. Heat loss in healthy individuals at rest is around $60-75 \mathrm{kcal} / \mathrm{h}$. In trauma patients heat loss can reach $400 \mathrm{kcal} / \mathrm{h}$. Administering opioids and muscle relaxants additionally accelerates hypothermia as these factors inhibit shivering and therefore thermogenesis [2]. The likelihood of post-traumatic hypothermia depends on the severity of injuries. In minor injuries, the incidence of post-traumatic hypothermia does not exceed 10\% [3], while among patients requiring intensive care unit treatment, it reaches almost 58\% [4]. Hypothermia increases the mortality rate by $20 \%$ in the immediate post-traumatic period [5]. In a study comparing the four-week survival rate of patients, the mortality rate of hypothermic patients is about $50 \%$ higher than in normothermic patients [6]. Patients with post-traumatic hypothermia also have a significantly higher demand for blood products, longer hospital stays and a greater risk of sepsis and multiorgan failure [5, 7-9]. A Tc drop is not only a sign that accompanies extensive trauma, but is an independent risk factor for mortality $[10,11]$.

The lack of awareness on the subject of heat loss increases the risk of hypothermia onset, especially in patients with severe trauma. The most common causes of heat loss are: insufficient thermal insulation, infusion of cold fluids, and ventilating with cold gases. A significant cause of iatrogenic hypothermia are prolonged surgeries. General anesthesia impairs thermoregulatory mechanisms while transfusions of cold fluids, as well as open body cavities expose internal organs to temperatures in the operating room $[1,6]$.

To date, Poland does not have written recommendations and criteria for treating post-traumatic hypothermia. There is also little data on readiness and inventory of equipment in Polish hospitals for the prevention and treatment of hypothermia [12].

The aim of this study was to assess the procedures and availability of equipment indispensable for diagnosing and treating post-traumatic hypothermia in ICUs, operating rooms, and adult surgical departments. This pilot study was conducted in the Holy Cross 
Province (1.25 million residents) which has similar climate conditions to its neighboring provinces, the number of injuries only slightly exceeds the national average (1006 admissions to hospital/100,000 residents/year) [13], and it has an even distribution of medical facilities.

\section{METHODS}

Survey forms were sent to the heads of all intensive care units and surgical departments in the Holy Cross Province, which receive injured patients transported to them by ambulance. General surgery departments were selected in the pilot study as they care for patients of interest through Poland's national health care system. Internal organ damage (chest and abdomen) and minor head injuries are treated by general surgery departments while injuries to the musculoskeletal system are treated by orthopedic departments. Thus, most patients with severe injuries who are at a high risk of developing post-traumatic hypothermia are mainly found in general surgery wards. The survey indicated that patients of interest were those who required ICU admission or needed an urgent laparotomy, thoracotomy or craniotomy immediately after admission to the hospital.

The survey consisted of 12 single-choice questions, however 4 questions included an open response option. The questionnaires were sent to all 14 adult general surgery departments and also to 13 ICUs located in the same hospitals. The survey asked about equipment used for measuring core temperature, locations and criteria for implementing temperature measurements, along with equipment for rewarming and criteria for implementing the rewarming of patients. The ICU survey additionally asked the same questions but pertaining to the perioperative period. Respondents were also surveyed about the scope of information entered into medical records regarding post-traumatic hypothermia. Consent of the bioethical commission was not deemed necessary since the study does not rely on data from patients' medical records. Due to the small sample size, the analysis is limited to statistical description. Data is presented with means of frequency and percentages.

\section{RESULTS}

Out of the 14 surgical departments, 13 completed the survey (93\%) and 12/13 ICUs returned completed surveys (92\%).

\section{Surgical Departments}

92\% (12/13) did not possess a thermometer for measuring core body temperature. One department claimed to measure Tc exclusively in patients who were admitted immediately from the emergency department (ED). Eleven (85\%) did not have the tools to rewarm patients. Two departments owned forced air convective warming devices and one of them also owned a conductive electric heating blanket. One of the departments initiated rewarming at $<35^{\circ} \mathrm{C}$ while the other proceeded after "clinical signs of hypothermia". Only one of the departments (8\%) had a standard operating protocol for monitoring Tc and procedures for rewarming. The majority of departments - 77\% (10/13) did not prewarm non-blood product infusions. One department owned a flow warmer and two departments used prewarmed bottles of solutions for infusion.

\section{Intensive Care Units}

All intensive care units had thermometers for measuring Tc. $58 \%$ (7/12) had esophageal probes and $42 \%(5 / 12)$ had rectal probes. Monitoring of Tc in every patient with severe trauma was accomplished in $17 \%$ of ICUs. In $8 \%$ of ICUs, Tc was monitored only in patients admitted from the operating room and in another $8 \%$ of ICUs only in patients admitted directly from the ED. Two wards (17\%) did not measure Tc at all. The remaining 50\% of ICUs followed their own self authored criteria for initiating Tc measurement such as: "patient history", "drop in skin temperature", "suspicion of hypothermia", and "discretion". In 67\% (8/12) of ICUs, Tc monitoring was started upon admission and in $17 \%(2 / 12)$ - at a later point but within 24 hours of admission. $83 \%$ of ICUs had equipment for warming patients $58 \%$ had convection devices (forced air), 33\% had electric blankets, and $17 \%$ used chemical heating blankets. None of the departments had the capability for warming gases in a respirator. One of the ICUs initiated warming routinely to all injured patients upon admission to the ward. While $50 \%(6 / 12)$ of the departments initiated warming of patients based on a Tc measurement, 3 of them began treatment with a reading of $<36^{\circ} \mathrm{C}$, and 3 departments warmed at $<35^{\circ} \mathrm{C}$. In $25 \%$ of the ICUs, warming was implemented on the basis of other criteria - "discretion", "interview, season of the year, coagulopathy", "severe trauma, symptoms of hypothermia". Most of the departments did not have standard operating protocols for measuring Tc (92\%) and for warming patients (83\%). 92\% of the ICUs (11/12) claimed to prewarm non-blood products for infusion, with 7/12 using flow-warmers and 4/12 preheating their solution containers before infusions.

\section{Operating Rooms}

During urgent surgical procedures after sustained trauma, 58\% (7/12) of the hospitals did not monitor Tc while 1 hospital routinely measured Tc and the remaining 4 (33\%) did so on an individual basis decided on by the anesthesiologist. Of the 5 hospitals that measure Tc, 4 used esophageal probes and the remaining hospital used rectal probes. All of the operating rooms were equipped with rewarming devices - 33\% had forced air systems, $25 \%$ had electrical blankets, $17 \%$ ventilated with warmed gases and $33 \%$ had other devices such as warming mattresses and chemical heating blankets (Fig. 1) However, 4 hospitals (33\%) did not warm patients in the operating room. Three hospitals initiated warming based on Tc - two hospitals began at $<36^{\circ} \mathrm{C}$ and at the remaining one at $\mathrm{Tc}<35^{\circ} \mathrm{C}$. Four hospitals did so on their own criteria: "symptoms of hypothermia", "length of operation", "discretion", "skin temperature, active hemorrhage, massive transfusions", "patient interview, season of the year, coagulopathy". Half of the ORs used flow warmers for infusing fluids, $42 \%$ conducted infusions with prewarmed containers, and one hospital (8\%) did not prewarm its fluids. Warming of patients during surgical procedures was recorded by the anesthesiologist in $17 \%$ of the hospitals and a Tc measurement was recorded in $25 \%$ of them.

\section{DISCUSSION}

Our study indicates that the majority of surgical departments in the Holy Cross Province are not properly equipped to diagnose and treat post-traumatic hypothermia. ICUs and operating rooms possess the proper equipment but lack unambiguous criteria and standard operating protocols for treating patients with hypothermia. 
The authors were unable to find any similar study pertaining to inventories of thermometers for measuring Tc or rewarming equipment in ICUs or surgical departments. Data of this kind has been found but in pre-hospital setting studies. Karlsen et al. and Henriksson et al. described the availability of this type of equipment in ambulances in Norway and Sweden $[14,15]$. Another study analyzed the equipment carried by mountain rescue services for treating victims of hypothermia [16].

The availability of thermometers and Tc measurement protocols in hospitals can be indirectly inferred from the percentage of patients who received such measurements. In the London trauma center, a screening arrival Tc was measured in $38 \%$ of patients [2]. Data from the national injury registers in the US and Germany showed that Tc is measured in $62 \%$ and $38.5 \%$ patients, respectively $[5,17]$. On the other hand, in the Ireland et al. study, only 2.7\% of patients admitted to the trauma center in Australia did not have deep temperature measured [7]. Reasons for the differences above, apart from the availability of equipment, may be hypothermia awareness and the level of staff training as well as the adopted recommendations and guidelines.

The differing Tc values below which ICU implement rewarming protocols reflect inconsistent views on the criteria for diagnosis of post-traumatic hypothermia. Perlman et al. indicate $36^{\circ} \mathrm{C}$ as the cut-off for diagnosing post-traumatic hypothermia, while Martin and Shafi accept $35^{\circ} \mathrm{C}$ as the cut-off in the National Trauma Data Bank analysis $[1,5,11]$. Meanwhile, $1 / 4$ of the ICUs do not have consistent criteria for implementing rewarming and $83 \%$ of ICUs do not have any standardized protocols for rewarming patients. Despite the well-known phenomenon of heat loss during surgery, $58 \%$ of the operating blocks in the Holy Cross Province do not monitor Tc, and $1 / 3$ of them do not rewarm patients. Meanwhile, lowering body temperature by only $1^{\circ} \mathrm{C}$ during surgery significantly increases blood loss [18]. Perioperative active warming was also found to reduce the rate of surgical wound infections and complications [19]. Although post-traumatic hypothermia is recognized as a risk factor for mortality [11], and its prevention is easy and low cost, $85 \%$ of the general surgery departments do not actively rewarm patients. Similarly, neither do $33 \%$ of operating blocks and $17 \%$ of ICUs. It is possible that these stem from limited knowledge of the impact of post-traumatic hypothermia on mortality. In a survey study conducted among doctors and nurses at trauma center in Australia, only $27 \%$ of respondents indicated coagulopathy as a possible consequence of hypother-

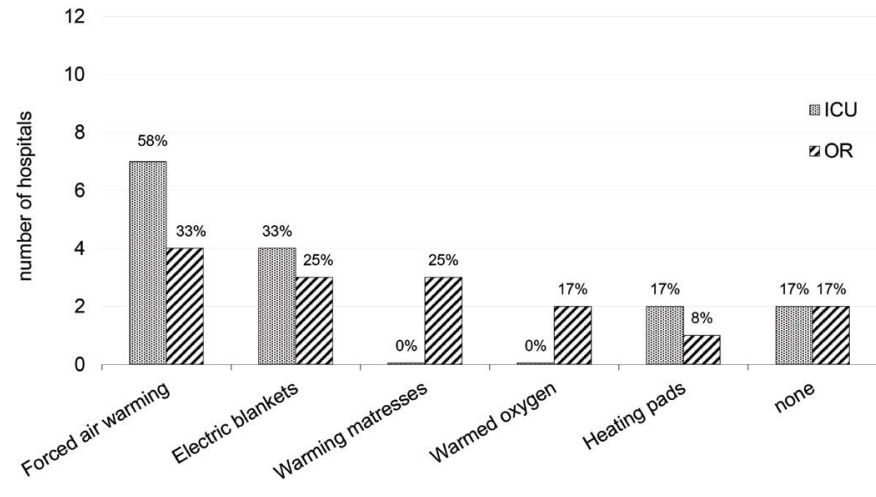

Fig. 1. Available equipment for rewarming patients in ICUs and ORs.

mia, and only $12.5 \%$ knew about the correlation of hypothermia with mortality in trauma [20]. In addition to supplying equipment, acknowledging the problem by forming an educational program along with criteria and standard operating protocols should be the next step. The results of our study point to some insufficiencies and failures in the health care system, but progress in this field will only occur after becoming aware of them. On the basis of this single study, the need for future actions is made clear. This is a multi-faceted process that requires awareness for improvements followed by creating local algorithms and assessing their results. This process is necessary for maximizing the effectiveness of the rescue system [21, 22].

\section{Limitations of the study}

The survey is a pilot study and results pertain to only one province in the country. However, health care system structure and law regulations are common in the whole country.

\section{CONCLUSIONS}

The majority of surgical departments in the Holy Cross Province are not prepared to handle post-traumatic hypothermia. Intensive care units are properly equipped but standardized protocols are lacking and equipment is not fully utilized. Prevention and treatment of intraoperative hypothermia in victims of injuries seems insufficient. These findings indicate a further need to continue this study but encompassing all of Poland while also taking into account pediatric surgical wards.

\section{REFERENCES}

1. Perlman R., Callum J., Laflamme C., Tien H., Nascimento B., Beckett A. et al. A recommended early goal-directed management guideline for the prevention of hypothermia-related transfusion, morbidity, and mortality in severely injured trauma patients. Crit Care. Critical Care, 2016; 20: 107.

2. Langhelle A., Lockey D., Harris T., Davies G.: Body temperature of trauma patients on admission to hospital: A comparison of anaesthetised and non-anaesthetised patients. Emerg Med J., 2012; 29: 239-242.

3. Soreide K.: Clinical and translational aspects of hypothermia in major trauma patients: From pathophysiology to prevention, prognosis and potential preservation. Injury. Elsevier Ltd, 2014; 45: 647-654.

4. Abelha F.J., Castro M.A., Neves A.M., Landeiro N.M., Santos C.C.: Hypothermia in a surgical intensive care unit. BMC Anesthesiol., 2005; 5: 1-10.

5. Martin R.S., Kilgo P.D., Miller P.R., Hoth J.J., Meredith J.W., Chang M.C.: Injury-associated hypothermia: An analysis of the 2004 National Trauma Data Bank. Shock, 2005; 24: 114-118.

6. Balvers K., Van der Horst M., Graumans M., Boer C., Binnekade J., Goslings Jc. et al.: Hypothermia as a predictor for mortality in trauma patients at admittance to the intensive care unit. J Emerg Trauma Shock, 2016; 9: 97.

7. Ireland S., Endacott R., Cameron P., Fitzgerald M., Paul E.: The incidence and significance of accidental hypothermia in major trauma - A prospective observational study. Resuscitation, 2011; 82: 300-306.

8. Arthurs Z., Cuadrado D., Beekley A., Grathwohl K., Perkins J., Rush R. et al.: The impact of hypothermia on trauma care at the 31st combat support hospital. Am J Surg, 2006; 191: 610-614.

9. Klauke N., Graff I., Fleischer A., Boehm O., Guttenthaler V., Baumgarten G. et al.: Effects of prehospital hypothermia on transfusion requirements and outcomes: a retrospective observatory trial. BMJ Open, 2016; 6: e009913.

10. Gentilello L.M., Jurkovich G.J., Stark M.S., Hassantash S.A., O'Keefe G.E.: Is hypothermia in the victim of major trauma protective or harmful? A randomized, prospective study. Ann Surg, 1997; 226: 439-449. 
11. Shafi S., Elliott A.C., Gentilello L.: Is Hypothermia Simply a Marker of Shock and Injury Severity or an Independent Risk Factor for Mortality in Trauma Patients? Analysis of a Large National Trauma Registry. J Trauma Inj Infect Crit Care, 2005; 59: 1081-1085.

12. Kosiński S., Darocha T., Gałązkowski R., Drwiła R.: Accidental hypothermia in Poland - estimation of prevalence, diagnostic methods and treatment. Scand J Trauma Resusc Emerg Med, 2015; 23: 1-6.

13. Health Needs Maps. Ministry of Health [Internet]. [cited 2018 Feb 15]. Available from: http://www.mpz.mz.gov.pl.

14. Karlsen A.M., Thomassen O., Vikenes B.H., Brattebo G.: Equipment to prevent, diagnose, and treat hypothermia: A survey of Norwegian pre-hospital services. Scand J Trauma Resusc Emerg Med., 2013; 21: 63.

15. Henriksson O., Bjornstig U., Saveman B.I., Lundgren P.J.: Protection against cold - a survey of available equipment in Swedish pre-hospital services. Acta Anaesthesiol Scand, 2017; 61: 1354-1360.

16. Podsiadło P., Darocha T., Kosiński S., Sałapa K., Ziętkiewicz M., Sanak T. et al.: Severe Hypothermia Management in Mountain Rescue: A Survey Study. High Alt Med Biol, 2017; 18: ham.2017.0090.
17. Weuster M., Bruck A., Lippross S., Menzdorf L., Fitschen-Oestern S., Behrendt P. et al.: Epidemiology of accidental hypothermia in polytrauma patients: An analysis of 15,230 patients of the TraumaRegister DGU. J Trauma Acute Care, Surg., 2016; 81: 905-912.

18. Rajagopalan S., Mascha E., Na J. et al.: The effects of mild perioperative hypothermia on blood loss and transfusion requirement. Anesthesiology, 2008; 108(1): 71-77.

19. Madrid E., Urrutia G., Roque i Figuls M., Pardo-Hernandez H., Campos J.M., Paniagua P. et al.: Active body surface warming systems for preventing complications caused by inadvertent perioperative hypothermia in adults. Cochrane database Syst Rev, 2016; 4: CD009016.

20. Ireland S., Murdoch K., Ormrod P., Saliba E., Endacott R., Fitzgerald M. et al.: Nursing and medical staff knowledge regarding the monitoring and management of accidental or exposure hypothermia in adult major trauma patients. Int J Nurs Pract, 2006; 12: 308-318.

21. Block J., Lilienthal M., Cullen L., White A.: Evidence-based thermoregulation for adult trauma patients. Crit Care Nurs Q, 2012; 35: 50-63.

22. Goniewicz K., Goniewicz M., Pawłowski W., Fiedor P., Lasota D.: Risk of road traffic accidents in children. Med Stud, 2017; 2: 155-160.

\begin{tabular}{llll}
\hline Word count: $2610 \quad$ Page count: 5 & Tables: - & Figures: 1 & References: 22
\end{tabular}

DOI: $\quad 10.5604 / 01.3001 .0013 .0146$

Table of content: https://ppch.pl/issue/11867

Copyright: Copyright @ 2019 Fundacja Polski Przegląd Chirurgiczny. Published by Index Copernicus Sp. z o. o. All rights reserved.

Competing interests: The authors declare that they have no competing interests.

The content of the journal „Polish Journal of Surgery" is circulated on the basis

- of the Open Access which means free and limitless access to scientific data.

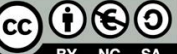

BY NC SA

This material is available under the Creative Commons - Attribution 4.0 GB. The full terms of this license are available on: http://creativecommons.org/licenses/by-nc-sa/4.0/legalcode

Corresponding author: Paweł Podsiadło, Polish Medical Air Rescue, ul. Jana Pawła ll 9a, 26-001 Masłów_; Tel. +48 222277605 ; E-mail: p.podsiadlo.01@gmail.com

Cite this article as:

Podsiadło P., Kosiński S., Darocha T., Zerebiec K., Wolak P., Kielczewski S., Plewa S., Głuszek S.: Diagnosis and treatment of post-traumatic hypothermia in hospitals - a pilot study; Pol Przegl Chir 2019: 91 (2): 24-28 
